\title{
The effects of the gender-culture interaction on self-reports of depressive symptoms: Cross-cultural study among Egyptians and Canadians.
}

\author{
Vivian Huang ${ }^{1}$, Shadi Beshai ${ }^{\text {Corresp.. }}{ }^{2}$, Mabel Yu ${ }^{2}$ \\ 1 Department of Psychology, Ryerson University, Toronto, Ontario, Canada \\ 2 Department of Psychology, University of Regina, Regina, Saskatchewan, Canada \\ Corresponding Author: Shadi Beshai \\ Email address: Shadi.Beshai@uregina.ca
}

Purpose: Research in depression has revealed differences in the way depressed individuals across cultures report their symptoms. This literature also points to possible differences in symptom reporting patterns between men and women. Using data from a larger dataset (Beshai et al., 2016), the current study examined whether non-depressed and depressed Egyptian and Canadian men and women differed in their self-report of the various domains of the Beck Depression Inventory - II (BDI-II). Method: We recruited a total of 131 depressed and non-depressed participants from both Egypt ( $n=29$ depressed; $n=29$ non-depressed) and Canada ( $n=35$ depressed; $n=38$ non-depressed). Depression status was ascertained using a structured interview. All participants were asked to complete the BDI-II along with other self-report measures of depression. BDI-II items were divided into two subscales in accordance with Dozois and Dobson's (1998) factor analysis: cognitive-affective and somatic-vegetative subscales. Results: We found a significant three-way interaction effect on the cognitive-affective $(F(1,121)=9.51, p=$ $.003)$ and main effect of depression status on somatic-vegetative subscales $(F(1,121)=$ $42.80, p<.001)$. Post hoc analyses revealed that depressed Egyptian men reported lower scores on the cognitive-affective subscale of the BDI-II compared to their depressed Canadian male counterparts. Conclusions: These results suggest that males across cultures may differentially report cognitive symptoms of depression. These results also suggest that clinicians and clinical scientists need to further examine the interaction effect of culture and gender when investigating self-reported symptoms of depression. 
1

2

3

4

5

The Effects of the Gender-Culture Interaction on Self-Reports of Depressive Symptoms:

7

8

9

10

11

12

13

14

15

16

17

\section{Cross-Cultural Study among Egyptians and Canadians}

Vivian Huang ${ }^{1}$, Shadi Beshai ${ }^{2}, \&$ Mabel $\mathrm{Yu}^{2}$

1. Department of Psychology, Ryerson University, Toronto, ON, Canada

2. Department of Psychology, University of Regina, Regina, SK, Canada

15 Corresponding author: Dr. Shadi Beshai, shadi.beshai@uregina.ca 
Abstract

19 Purpose: Research in depression has revealed differences in the way depressed individuals across cultures report their symptoms. This literature also points to possible differences in symptom reporting patterns between men and women. Using data from a larger dataset (Beshai et al., 2016), the current study examined whether non-depressed and depressed Egyptian and Canadian men and women differed in their self-report of the various domains of the Beck Depression Inventory - II (BDI-II). Method: We recruited a total of 131 depressed and nondepressed participants from both Egypt ( $n=29$ depressed; $n=29$ non-depressed) and Canada ( $n$ $=35$ depressed; $n=38$ non-depressed). Depression status was ascertained using a structured interview. All participants were asked to complete the BDI-II along with other self-report measures of depression. BDI-II items were divided into two subscales in accordance with Dozois and Dobson's (1998) factor analysis: cognitive-affective and somatic-vegetative subscales.

Results: We found a significant three-way interaction effect on the cognitive-affective subscale $(F(1,121)=9.51, p=.003)$ and a main effect of depression status on somatic-vegetative subscale $(F(1,121)=42.80, p<.001)$. Post hoc analyses revealed that depressed Egyptian men reported lower scores on the cognitive-affective subscale of the BDI-II compared to their depressed Canadian male counterparts. Conclusions: These results suggest that males across cultures may differentially report cognitive symptoms of depression. These results also suggest that clinicians and clinical scientists need to further examine the interaction effect of culture and gender when investigating self-reported symptoms of depression. 


\section{Introduction}

Cross-cultural epidemiological data indicate that depression is prevalent in almost all regions of the world (Kessler \& Bromet, 2013). Specifically, epidemiological studies carried out in countries such as Lebanon (Karam et al., 2006), Morocco (Kadri et al., 2010), Saudi Arabia (Desouky et al., 2015), and Egypt (Ghanem et al., 2009) reveal that depression is a prevalent condition in the Arab region. Depression carries an enormous global burden (Murray \& Lopez, 1997). Therefore, studies that attempt to elucidate the nature and features of this condition worldwide are necessary. Cross-cultural research in the field of depression has revealed important culture-related differences in the experience and presentation of the disorder. For example, a number of early studies found that non-Western individuals suffering from depression may present with somatic features of the condition (e.g., appetite and sleep disturbance, agitation, etc.; Ryder, Yang, \& Heine, 2002). However, relatively more recent evidence suggests that Western patients specifically may "psychologize" their presentation of depression. In other words, the difference in depressive symptom presentations between Western and non-Western individuals may be largely driven by Westerners' unique emphasis upon cognitive symptoms (e.g., feeling of sadness and anhedonia, negative thoughts about self, etc.; Kalibatseva \& Leong, 2011; Parker, Cheah, \& Roy, 2001; Ryder, Yang, Zhu, Yao, Yi, Heine, \& Bagby, 2008). Due to this differential emphasis across cultures, this study attempted to examine differences between depressed Canadians and Egyptians in self-reported somatic and cognitiveaffective symptoms of depression.

Somatization of depression has been observed in a number of cultures around the world.

For example, South Asian immigrants to the United States are less likely than Americans of European decent to recognize and label a vignette based on emotional features as depression 
64 (Karasz, 2005). Similarly, Koss-Chioino (1999) found that Puerto Rican patients of depression

65

66

67

68

generated fewer and less varied reports of cognitive symptoms in comparison to their reports of somatic symptoms.

In support of the somatization hypothesis among Middle-Eastern sufferers, Okasha (1999) indicated that, in describing their symptoms, depressed individuals in the Arab region reported shortness of breath, agitation, and other physiological changes. Further, a factor analytic study of the Centre for Epidemiologic Studies - Depression Scale (CES-D; Radloff, 1977) found that somatic and affective items of the scale, which are typically represented by different factors among Westerners, were fused in one factor among a sample of Egyptian students (Beshai, Dobson, \& Adel, 2013). El-Rufaie and colleagues (1999) found that depression and anxiety were both significantly related to somatic complaints, such as abdominal pain, among their Saudi Arabian samples. Further, Farhood and Dimassi (2012) found that depression, anxiety, and somatic complaints were significantly associated among a sample of Lebanese citizens experiencing war related post-traumatic stress symptoms. Finally, Simon and colleagues (1999) found that $51 \%$ of patients with depression had unexplained somatic symptoms, while $5 \%$ of this depressed sample denied the psychological symptoms of their depression.

By contrast, some recent evidence suggests that the concept of somatization should not be over-applied, and that this tendency is not entirely unique to non-Westerners. For example, somatization is often emphasized in Chinese populations due to the different cultural interpretations of the importance and/or the acceptability of somatic and psychological symptoms (Ryder, Yang, \& Heine, 2002). However, this characterization is not limited to the Chinese culture as evidenced by the findings of Zhou and colleagues (2011), who found that 
86

87

88

89

90

91

92

93

94

95

96

97

anxious Euro-Canadian participants were more likely than their Han Chinese counterparts to somatize their anxiety symptoms.

Further, emerging evidence suggests that the somatic emphasis in most regions of the world may be due to differences in symptom reporting patterns, as opposed to the nature of the disorder in these regions. For example, immigrant women from South Asia who lived in England recognized that depression is associated with both cognitive and somatic symptoms, but they emphasized the latter since they perceived them as more legitimate (Burr \& Chapman, 2004). In Arab nations, cognitive symptoms, such as guilt, may be associated with "sin" in Islam, and thus their presence may imply moral culpability (Beshai, Clark, \& Dobson, 2013; El-Islam, 1969). Further, Goldberg and Bridges (1988) argued that somatization is a way for psychologically distressed individuals to assume the sick role without the stigma of mental illness. Past findings show that the stigma surrounding depression is more severe among Chinese Americans than European Americans (Hsu et al., 2008). As such, Chinese populations may present somatic symptoms more readily than Western populations in order to avoid psychiatric stigma.

Further, the distinction between symptom experience and reporting is not as straightforward as previously thought. Recent findings suggest that culture plays an important role in symptom presentation (e.g., Dere et al., 2013). For example, since the Chinese culture encourages a focus away from internal experiences (e.g., emotions) and towards concrete details of the external world, Chinese patients may perceive somatic symptoms as more salient than cognitive symptoms (Ryder \& Chentsova-Dutton, 2012). In this regard, somatization is a culturally shaped style for expressing distress. Additionally, linguistic factors also contribute to the differential emphasis of somatic and cognitive symptoms across cultures. Although there are words to describe psychological states in the Chinese language, somatic metaphors are part of 
109 the communication style of the culture and thereby often used to express distress (Lee \&

110 Kleinman, 2007; Leff, 1988). Similarly, Hamdi, Amin, and Abou-Saleh (1997) have suggested

111 that the emphasis on somatic features among Arab sufferers of depression may be due to the lack

112 of available linguistic idioms to express cognitive and affective symptoms in this region. As

113 such, it is possible that translated Western questionnaires and other diagnostic instruments

114 administered to participants in this region may provide a lexicon for such idioms.

115 Thoughts about self and future have often been associated with cognitive-affective

116 symptoms of depression (Beshai, Clark, \& Dobson, 2012; Beshai, Dobson, Adel, \& Hanna,

117 2016). Therefore, negative self-referent thoughts have often been believed to be absent among

118 depressed individuals of non-Western, and particularly Arabic origins (reviewed in Beshai et al.,

119 2012). Inconsistent with such beliefs, Beshai and colleagues (2012; 2016) found that Egyptian

120 individuals showing signs of depression reported similar levels of negative and positive thoughts

121 about the self when compared with their Canadian counterparts.

122 Methodological artifacts may also explain some of findings in the literature regarding

123 differences in self-reported depression symptoms across cultures. For example, some research

124 suggests that individuals of Arabic descent express cognitive and affective symptoms of

125 depression more readily when the mode of assessment takes the form of paper-and-pencil self-

126 reports (Matthey, Barnett, \& Elliot, 1997). In addition, some evidence suggests that nonverbal

127 reinforcement of cognitive-affective versus somatic responses by clinicians may be related to the

128 preferential reporting of cognitive-affective symptoms in Western countries (Lam, Marra, \&

129 Salzinger, 2005). As Chentsova-Dutton and Tsai (2008) argued, both somatic and cognitive

130 features of depression are part of the disorder across cultures, and so the cultural emphasis of one

131 set of symptoms over the other may reflect preferential reporting and comfort of disclosure rather 
132 than the experience itself. Although as mentioned above, it is difficult to disentangle symptom 133 reporting from experience.

134 The majority of research in this area has investigated somatic and cognitive symptoms in 135 broad categorical sets, rather than individual symptoms; however, recent findings highlight the 136 need for a more nuanced examination (Dere et al., 2013). Parker, Cheah, and Roy (2001)

137 examined individual somatic and cognitive depression symptoms to determine the differences 138 between Malaysian and Australian samples on the severity threshold for reporting certain 139 depression symptoms. They found that although the actual prevalence of reporting "health 140 problems" was comparable between the two groups (76\% for Malaysian and 66\% for Australian), the threshold at which the Malaysian sample reported this item was much lower than that of their Australian counterpart. Similarly, the severity threshold for reporting "chest pain" was also lower in the Malaysian sample, whereas the Australian sample reported such a symptom only when it was relatively severe. Studying symptoms at the individual level rather than amalgamating them into broad categorical sets may allow for a more nuanced understanding of the distinction between somatic and cognitive symptoms.

Recent findings by Dere et al. (2013) also attempted to address culture variations in symptom presentation through a more nuanced fashion. The researchers examined individual somatic and cognitive symptoms and found that certain symptoms deviated from their overall symptom reporting pattern. Despite reporting lower levels of cognitive symptoms compared to

151 Euro-Canadian participants, the Chinese sample endorsed higher levels of affective symptoms 152 (depressed mood, suppressed emotions) relative to their overall trend of reporting. Moreover, 153 contrary to the popular assumption that Chinese are more reluctant to discuss depression, the 154 Chinese and Euro-Canadian samples reported "depressed mood" at similar levels (56\% and 
$15548.6 \%$, respectively). Further, and inconsistent with previous findings that Chinese outpatients

156 endorsed significantly greater levels of somatic symptoms compared to Euro-Canadian

157 outpatients (Ryder et al., 2008), Dere et al. (2013) found that Euro-Canadian participants had

158 significantly greater levels of atypical somatic symptoms - appetite gain, weight gain, and

159 hypersomnia - than their Chinese counterpart. Although the literature provides substantial

160 evidence for non-Western emphasis on somatization and Western emphasis on psychologization,

161 these findings suggest that this generalization may not hold true across all symptoms.

162 In addition to cross-cultural differences, there are possible gender differences in the

163 experience of depressive symptoms. Consistent findings suggest that women are twice as likely

164 as men to experience depressive symptoms in their lifetime (Angst et al., 2002; for review, see

165 Kuehner, 2003). Further, these gender differences in the prevalence of depression appear to be

166 consistent across cultures (Maier, Gansicke, Gater, Rezaki, Tiemens, \& Urzua, 1999). Previous

167 studies have elucidated various underlying factors that may contribute to gender differences in

168 depression rates, which include cognitive (e.g., Nolen-Hoeksema \& Jackson, 2001), biological

169 (e.g., Stalye et al., 2006; Moieni et al., 2015), neurological (e.g., Yao et al., 2014), and social

170 (e.g., Dalgard et al., 2006) factors. Consistent with literature from the West, a review by Eloul

171 Ambusaidi, and Al-Adawi (2009) found heightened rates of depression among women in Middle

172 Eastern and North African countries compared to men.

173 In addition to differences in prevalence, one study suggested that females tend to report

174 more somatic and less cognitive symptoms of depression and when compared with their male

175 counterparts (e.g., Schuch, Roest, Nolen, Penninx, \& de Jonge 2014). Specifically, women

176 appear to more frequently report changes in appetite, sleep disturbance, psychomotor retardation,

177 negative perception of physical health, and greater functional impairment than do men 
178 (Kornstein et al., 2000; Marcus et al., 2005; Silverstein, 2002). Although some cross-cultural

179 studies have examined different symptom reporting across genders, the focus has mainly been on

180 females and somatization of symptoms. Very few cross-cultural studies to date have examined

181 gender differences in reporting both cognitive and somatic depressive symptoms. Thus, studies

182 that examine cross-cultural and cross-gender differences in symptom reporting in depression

183 may be worthwhile.

184 Understanding differential experiences and reporting of symptoms across cultures is

185 important, as this understanding may aid in tailoring effective treatments for individuals of

186 varying cultural groups. For example, in cognitive therapy for depression (Beck, Rush, Shaw, \&

187 Emery, 1979), which is one of the most used and evaluated psychotherapies for the disorder, a

188 significant portion of therapeutic time and effort is spent tackling "cognitive" features of the

189 disorder (negative automatic thoughts, attitudes, etc.; Beck et al., 1979). Accordingly, if these

190 symptoms are not routinely reported or if they do not significantly correlate with meaningful

191 depressive experience among people of Arabic descent, then cognitive therapy's dedication to

192 dismantling negative cognitions may be unwarranted among these patients. Further, examining

193 the effects of the culture-gender interaction on self-reported symptoms of depression may aid in

194 the appropriate and more nuanced assessment and diagnosis of this disorder in various cultures.

195 The current study attempted to close the gaps in the current literature by examining the

196 frequency and relationships of somatic and cognitive-affective symptoms of depression among a

197 group of depressed Egyptian patients. The data for this study were collected as part of a larger

198 trial (Beshai, Dobson, Adel, \& Hanna, 2016) that examined the cognitive model of depression in

199 Egypt. In the current study, we computed separate scores for the somatic-vegetative and

200 cognitive-affective domains of the Beck depression Inventory-II (BDI-II; Beck, Steer, \& Brown, 
201 1996), and compared these cross-culturally (Egyptians and Canadians), across genders, and

202 across depression statuses (depressed and non-depressed). In accordance with earlier studies on

203 somatization among Egyptians (e.g., Okasha, 1999), we hypothesized a culture by depression

204 status interaction on the somatic-vegetative subscale of the BDI-II, wherein depressed Egyptians

205 would exhibit higher scores on this subscale than depressed Canadians. Given that the literature

206 suggests Westerners tend to "psychologize" their depression (Ryder et al., 2002), we

207 hypothesized a second culture by depression status interaction, such that, in comparison to

208 depressed Egyptians, depressed Canadians would show higher scores on the cognitive-affective

209 subscale of the BDI-II. We did not anticipate a three-way interaction.

\section{Method}

Data from a total of 58 Egyptians (29 depressed and 29 non-depressed) and 73 Canadians

212 (35 depressed and 38 non-depressed) were used for this study. The data were collected in the

213 context of a larger trial by Beshai and colleagues (2016). Depressed participants were either

214 recruited from a psychiatric clinic (Cairo, Egypt), or from the community via poster notices and

215 social media (e.g., Facebook) advertisement. Non-depressed Egyptian participants were recruited

216 from the community at large, whereas non-depressed Canadians were recruited via recruitment

217 posters or social media outlets that identified the target sample (e.g., individuals with depression)

218 in the community of Calgary, Alberta, Canada. A power analysis was conducted to estimate the

219 appropriate sample size for the current study. Using G*Power (Faul, Erdfelder, Lang, \&

220 Buchmer, 2007), a minimum sample size of 111 was needed to detect a medium-large effect

221 (Cohen's $f=0.35$; with a priori $\alpha=.05, \beta=.80$ ) based on the current $2 \times 2 \times 2$ design.

To determine eligibility of potential participants, study researchers administered a

223 bespoke structured interview (Structured Interview Screen, described below) designed to 
224 ascertain the presence of depressive symptoms and absence of other exclusionary diagnostic

225 criteria (e.g., mania, psychosis, substance dependence). All depressed participants in both

226 countries exhibited symptoms in accordance with DSM-IV-TR (APA, 2002) Major Depressive

227 Episode criteria. All non-depressed participants showed no diagnostic criteria for bipolar

228 disorder and other aforementioned exclusionary diagnoses, and had no previous history of major

229 depression. Eligible participants were then invited to the clinic/lab to participate in the study.

230 Upon arrival, all participants provided written informed consent and completed a questionnaire

231 package, which included demographic information and the BDI-II (Beck, Steer, \& Brown,

232 1996). Depression status was ascertained through responses on the initial structured interview, a

233 score of 14 or higher on the BDI-II, and a score of 9 or higher on the Psychiatric Diagnostic

234 Screening Questionnaire Depression Subscale (Zimmerman \& Mattia, 2011). The study was

235 approved by the Conjoint Faculties Research Ethics Board (CFREB) at the University of Calgary

236 (Approval reference: REB-6899).

237 To be included, participants reported that Egypt (for the Egyptian sample) or Canada (for

238 the Canadian sample) is the country of birth of self and parents, and that they were at least third

239 generation Egyptian (Egyptian sample) or third generation European (Canadian sample). Finally,

240 participants with symptoms of psychosis, mania, and substance dependence were excluded from

241 the study.

The Structured Interview Screen was initially administered to the Canadian and Egyptian

243 samples to determine the eligibility of participants. The screen consists of 14 questions, 5 of

244 which were pertaining to current depressive symptoms. The remaining items assessed symptoms

245 related to diagnostic (i.e., psychosis, mania, and substance dependence) and demographic (e.g.,

246 country of birth) exclusionary criteria. The items were constructed based on the Structure 
247 Clinical interview for the DSM-IV (SCID; First, Spitzer, Gibbon, \& Williams, 1997) and the

248 Composite International Diagnostic Interview 3.0 (Kessler \& Ustun, 2004). The depression items

249 had good reliability $(\alpha=.88)$ within the Canadian sample. Moreover, Canadian participants who

250 reported "Yes" to the interview question "In the last month, did you feel sad or depressed for

251 most of the day, nearly everyday" scored significantly higher on the BDI-II than those who

252 answered "No" to this question (BDI-II: $F(2,82)=115.22, p<.001)$. Accordingly, this

253 screening interview appeared to have good reliability and appeared consistent with other

254 depression measures in differentiating between depressed and non-depressed participants.

255 The Beck Depression Inventory - II (BDI-II; Beck et al., 1996) is a 21-item self-report

256 measure that evaluates the severity of depressive symptoms. The scale contains various domains

257 of the disorder, which include affective, cognitive, somatic, and motivational domains. Items are

258 answered on a 4-point Likert scale (ranging from 0 to 3), with a higher score on the scale

259 indicating greater severity of depressive symptoms. In order to operationalize the somatic and

260 cognitive symptom domains of depression, previous studies have conducted exploratory and

261 confirmatory factor analyses on the BDI-II to identify its latent factors (Dozois, Dobson \&

262 Ahnberg, 1998; Steer, Ball, \& Ranieri, 1999; Whisman, Perez, \& Ramel, 2000). Specifically, a

263 confirmatory factor analysis conducted by Dozois and colleagues (1998) suggested that items 1,

$2642,3,5,6,7,8,9,13$, and 14 were representative of the cognitive-affective domain and items 4 ,

$26510,11,12,15$ to 21 were representative of the somatic-vegetative domain of the BDI-II within a

266 college sample. Furthermore, Dozois and colleagues found that the two latent factors of the BDI-

267 II were highly correlated (Dozois et al., 1998). The current study calculated somatic-vegetative

268 and cognitive-affective symptom subscales in accordance with domains identified by Dozois et 
269 al. (1998). A series of ordinal logistic regressions were conducted to establish cross-cultural

270 equivalence of the BDI-II and the aforementioned subscales (See detailed analysis plan below).

All the materials used in Egypt were translated according to the World Health

272 Organization's guidelines. Accordingly, two professional translators were involved in the translation process of study material: one translator who forward translated the English materials into Arabic, and then the other who back translated the Arabic translation back to English. was used to determine statistical significance. In order to establish scalar equivalence, a series of ordinal logistic regressions were conducted to assess the uniform and non-uniform differential item functioning across the two ethnicities of participants. The analyses were conducted separately for the BDI-II total score, the BDI-II cognitive-affective and somatic-vegetative subscale scores. Following Zumbo (1991) and Slocum, Gelin, and Zumbo (2003), each item was entered as the independent variable. Dependent variables were entered in three separate steps: 1) total score, 2) cultural group variable, 3 ) total score $\times$ cultural group. Two criteria were set to identify differential functioning of items: a) significant two-degree-of-freedom chi-square test ( $p$ $<.01$ ), and/or b) a change in Nagelkerke $R^{2}$ of .13 or larger between Steps 1 and 3 (uniform differential functioning), and between Steps 2 and 3 (non-uniform differential functioning). If an item displayed differential functioning, it was removed from subsequent analyses. samples were assessed using one-way analysis of variance (ANOVA) and chi-square analysis. 
292 somatic-vegetative and cognitive-affective symptoms, as well as the total score of the BDI-II

293 within each subsample (i.e., Egyptian vs. Canadian). Two, three-way analyses of covariance

294 (ANCOVAs) were conducted in order to test cultural, gender, and depression status differences

295 on the BDI-II somatic-vegetative and cognitive-affective domains, while controlling for the other

296 subscale score. Finally, independent samples t-tests were conducted post hoc to delineate any

297 significant interactions found in the ANCOVA analyses.

298 Results

299 Participant characteristics

300 Participant had a mean age of 33.94 years $(S D=13.37$; range $=18-65)$. See Table 1 for

301 detailed sample characteristics. A one-way ANOVA revealed that Canadians were significantly

302 older than their Egyptian counterparts, $F(1,129)=8.83, p=.004$. The chi-square analyses

303 revealed that there were significant differences in economic status $\left(\chi^{2}[5]=13.28, p=.02\right)$ and

304 education level $\left(\chi^{2}[8]=21.11, p=.007\right)$ between the Egyptian and Canadian samples. Age was

305 also found to significantly correlate with other depression-related constructs (e.g., hopelessness;

306 Beshai et al., 2016). Accordingly, age was used as a covariate in subsequent ANCOVAs.

307

Insert Table 1 near here

308

309

310

311

312

313

314

\section{Cross-cultural equivalence of the BDI-II}

The three-step ordinal logistic regression analyses revealed that items 6 (Punishment feelings) and 11 (Agitation) displayed significant differential functioning. Specifically, the analyses revealed that item 6 showed significant 2 -df chi-square on total BDI-II scores $(\chi 2=$ 21.56, $p<.001 ; \Delta$ Nagelkerke $\left.R^{2}=0.13\right)$ and on the cognitive-affective subscale $(\chi 2=19.91, p<$ $.001 ; \Delta$ Nagelkerke $R^{2}=0.12$ ). Similarly, item 11 displayed significant chi-square on total BDI-II scores $\left(\chi 2=12.74, p=.002 ; \Delta\right.$ Nagelkerke $\left.R^{2}=0.06\right)$ and on the somatic-vegetative subscale $\left(\chi^{2}\right.$ 
$315=9.66, p=.007 ; \Delta$ Nagelkerke $\left.R^{2}=0.03\right)$. These items were removed from subsequent analyses.

316 The remaining 19 items of the BDI-II evidenced cross-cultural scalar invariance, and thus were

317 retained in calculating total and subscale scores. The final BDI-II total and subscale scores

318 displayed excellent internal consistencies among Egyptian (total: $\alpha=.93$; cognitive-affective: $\alpha$ $319=.88$; somatic-vegetative: $\alpha=.90$ ) and Canadian samples (total: $\alpha=.97$; cognitive-affective: $\alpha=$ 320.94 ; somatic-vegetative: $\alpha=.95)$.

321 Cross-national, gender, and depression status differences on total BDI-II scores

A Three-way ANOVA was conducted to examine cross-national, gender, and depression status differences on total BDI-II scores. The analysis revealed a significant nationality by depression status interaction $\left(F(1,123)=9.87, p=.002\right.$, partial $\left.\eta^{2}=.07\right)$, whereby depressed

325 Canadians reported higher total scores on the BDI-II compared to depressed Egyptians. Among non-depressed participants, there was no significant difference between nationalities on total scores of the BDI-II. There was also no significant nationality by gender interaction evidenced in the current analysis $(F(1,123)=1.79, p=.18)$, nor a gender by depression status interaction $(F(1,123)=1.53, p=.22)$. However, a significant main effect of gender on total BDI-II scores was revealed, $F(1,123)=5.76, p=.02$, partial $\left.\eta^{2}=.05\right)$. Specifically, females reported significantly higher total BDI-II scores than did males. positively correlated with the BDI-II somatic-vegetative symptoms among Egyptians $(r(57)=$ $.94, p<.001)$ and Canadians $(r(72)=.98, p<.001)$. Similarly, scores on the BDI-II cognitiveaffective subscale were significantly and positively correlated with total BDI-II scores among

337 Egyptians $(r(57)=.93, p<.001)$ and Canadians $(r(72)=.98, p<.001)$. Scores on the BDI-II 
338 somatic-vegetative and cognitive-affective subscales were significantly and positively correlated

339 with each other among Egyptians $(r(57)=.76, p<.001)$ and Canadians $(r(72)=.92, p<.001)$.

340 However, a Fisher's r-to-z transformation analysis revealed a stronger relationship between the

341 somatic-vegetative and cognitive-affective subscales among Canadians than among Egyptians

342 (Fisher's $z=3.29, p=.001)$.

343 Nationality, gender, depression status and cognitive-affective subscale scores

344 The three-way, 2 (Egyptian vs. Canadian) by 2 (Female vs. Male) by 2 (Depressed vs.

345 Non-depressed) ANCOVA was conducted in order to examine cross-cultural, gender, and

346 depression status differences on the BDI-II cognitive-affective and somatic-vegetative subscales,

347 using age and either subscale score as covariate when analyzing the other as the dependent

348 variable. The first analysis revealed a significant three-way interaction on the cognitive-affective

349 subscale of the BDI-II, $F(1,121)=9.51, p=.003$, partial $\eta^{2}=.07$ (See Fig. 1). This analysis also

350 evidenced a significant depression status by nationality interaction $(F(1,121)=4.87, p=.03$,

351 partial $\left.\eta^{2}=.04\right)$ and a significant nationality by gender interaction $(F(1,121)=8.047, p=.005$,

352 partial $\left.\eta^{2}=.06\right)$ on the cognitive-affective subscale.

353 As planned, follow-up independent sample t-tests were used to tease apart the significant

354 interaction found in the cognitive-affective domain of the BDI-II. Using Bonferroni corrections,

355 the alpha level was corrected to .01 to account for family-wise error rate (FWER). The tests

356 revealed that depressed Canadian males $(M=14.33, S D=5.26)$ reported significantly higher

357 scores on the cognitive-affective subscale of the BDI-II compared to depressed Egyptian males

$358(M=8.25, S D=4.24), t(22)=3.13, p=.005$. There was a marginally significant cross-national

359 difference in cognitive-affective symptoms of the BDI-II among non-depressed females.

360 Specifically, non-depressed Egyptian females $(M=2.58, S D=1.94)$ exhibited greater cognitive- 
361 affective symptoms on the BDI-II than their Canadian counterpart $(M=0.93, S D=1.53)$,

$362 t(27.34)=-2.27, p=.03$. There were no significant differences between depressed Egyptian and

363 Canadian females $(t(38)=-0.32, p=.75)$, and non-depressed Egyptian and Canadian males

$364(t(12.86)=-1.99, p=.07)$.

365

Insert Figure 1 near here

366

Nationality, gender, depression status, and somatic-vegetative subscale scores

367

Controlling for age and the cognitive-affective subscale scores, the ANCOVA revealed a

368

marginally significant three-way interaction of the somatic-vegetative subscale of the BDI-II,

$369 F(1,121)=3.28, p=.07$. Furthermore, there was a significant main effect of depression status

$370\left(\mathrm{~F}(1,121)=42.80, p<.001\right.$, partial $\left.\eta^{2}=.03\right)$. By examining the means, depressed individuals $(M$

$371=14.51, S D=4.91)$ reported significantly higher scores on the somatic-vegetative subscale

372 compared to their non-depressed counterparts $(M=1.60, S D=2.02)$.

373 Discussion

374 This study examined cross-cultural and gender differences on BDI-II subscale scores

375 (somatic-vegetative and cognitive-affective symptoms) among depressed and non-depressed

376 individuals. The results obtained in this study stress the need to examine depressive symptoms in

377 non-Western cultures as a multifaceted construct. Further, the results indicated that men and

378 women with different depression statuses in different cultures may report a different pattern of

379 symptoms. Contrary to the hypothesis, there were no significant two-way interaction between

380 nationality and depression status on the somatic-vegetative subscale of the BDI-II. However,

381 there was a significant three-way interaction, wherein depressed Egyptian males reported less

382 cognitive-affective symptoms than depressed Canadian males, after controlling for age and 
383 somatic-vegetative subscale scores. The current findings provided some new insight into cultural

384 differences in depressive symptom presentation between Egyptians and Canadians.

385 This study also examined the scalar invariance of the BDI-II across cultures. These

386 equivalence analyses revealed that, with the exception of the Punishment feelings (6) and

387 Agitation (11) items, the majority of BDI-II items evidenced scalar equivalence across cultures.

388 This suggests that the employed Arabic translation of the BDI-II was a valid measure of

389 depression amongst this Egyptian sample. Previous studies have also found measurement

390 invariance of the BDI-II in other cultures, such as Chinese (Byrne et al., 2007; Whisman et al.,

391 2012), Turkish (Canel-Cinarbas, Cui, \& Lauridsen, 2011), and South African (Makhubela, 2016)

392 among clinical and non-clinical samples. Given that this is one of the first studies to examine

393 scale equivalence of the Arabic version of the BDI-II, future studies are needed to replicate the

394 current findings using a larger sample, especially among depressed Egyptians. Future studies

395 also need to explore other indicators of scale invariance (e.g., configural and metric invariance;

396 Meredith, 1993).

397 Previous studies have suggested that non-Western cultures tend to emphasize somatic as

398 opposed to cognitive, symptoms in relation to their depression (Chentsova-Dutton \& Tsai, 2008;

399 Kosss-Chioino, 1999). This emphasis upon somatic complaints as key features of depression has

400 also been found among South Asian immigrants (Karasz, 2005), and African-American patients

401 (Sellers, Ward, \& Pate, 2006). Even in Egypt, some research has found that depressed

402 individuals in that region of the world tend to emphasize somatic complaints (Okasha, 1999;

403 Sami \& El-Gawad, 1995). However, the present study found no significant differences on self-

404 reports of somatic symptoms between depressed Egyptians and Canadians, after controlling for

405 age and the cognitive-affective subscale scores. As suggested by Dere et al., (2013), the non- 
406 Western emphasis upon somatization should not be over generalized. However, it is possible that

407 the current sample may not be fully representative of the population of depression sufferers in

408 Egypt. Particularly, all depressed Egyptian participants were recruited from a psychiatric clinic

409 in an affluent neighbourhood in Cairo, Egypt. Thus, these treatment-seeking individuals may

410 experience less self-stigma and may have greater acceptance of their condition, which in turn

411 may explain their relatively diminished emphasis on somatic symptoms. Furthermore, depressed

412 Egyptians may also have experienced less depressive symptoms overall, as this was reflected in

413 total BDI-II scores. As suggested by Ryder et al. (2008), more research is needed to examine the

414 effect of help-seeking strategies on symptom presentation. Future studies should recruit

415 Egyptians with depression via different avenues and/or methods to ensure the accurate

416 representation of depression in this region of the world. Nonetheless, the present findings may

417 shed light on depressive symptom endorsement between depressed Egyptians and European

418 Canadians.

419 We hypothesized that depressed Canadians would exhibit significantly higher scores on

420 the cognitive-affective subscale of the BDI-II. Partially consistent with our hypothesis, the

421 present study found a significant three-way interaction on the cognitive-affective subscale of the

422 BDI-II. Specifically, it was found that depressed Egyptian males reported significantly less

423 cognitive-affective complaints in comparison to depressed Canadian males. Consistent with

424 previous findings, Western individuals may emphasize psychological features of depression (i.e.,

425 greater emphasis on the cognitive-affective symptoms) compared to individuals of non-Western

426 descent (e.g., Kalibatseva, Leong, \& Ham 2014; Parker, Chaeh, \& Roy, 2001; Ryder et al.,

427 2008). However, this pattern of results also suggests differential symptom expression of

428 depression among males in particular. Although there are limited studies that have attempted to 
429 examine the experience and expression of depression in men, the few studies that have been 430 conducted suggest that male presentation might not map well onto existing diagnostic tools or 431 self-report measures of depression (e.g., Cochran \& Rabinowitz, 2000; for review see Addis, 432 2008). Moreover, stigma and gendered sociocultural norms in Egypt may have defined how men 433 should think and behave. In turn, these norms may shape how men respond to the experience of 434 depression and how they may express its symptoms (Addis \& Cohane, 2005). Considering Egypt 435 is a relatively conservative country (Okasha, 1999), it is possible that Egyptian males are 436 adherent to these sociocultural and gender norms compared to their Canadian counterparts. 437 In addition to gender and cultural norms, linguistic differences between Arabic and 438 English may result in different interpretations of the questionnaire items, especially cognitive 439 items (Hamdi, Amin, \& Abou-Saleh, 1997). However, due to the paucity of research on 440 depression and males specifically, it is difficult to identify specific factors that may contribute to 441 the current findings.

442 Overall, females reported greater total depressive symptoms compared to their male 443 counterparts. Consistent with previous findings, females are at greater risk of experiencing 444 depressive symptoms due to various underlying factors (e.g., Dalgard et al., 2006; Moieni et al., 445 2015). Further, we did not find any gender by depression status interaction on total BDI-II 446 scores. It appeared that, regardless of depression status, females tend to report greater overall 447 depressive symptoms in the current sample. Contrary to our hypothesis, we found a significant 448 nationality by gender interaction on the cognitive-affective subscale, wherein non-depressed 449 Egyptian females reported higher scores on this subscale compared to their Canadian 450 counterparts. Although differences were found, the mean cognitive-affective scores of the non451 depressed Egyptian females were below the cut-off for minor depression (Alansari, 2006). The 
452 relatively elevated scores among non-depressed Egyptian females may be contributed to daily or

453 social stressors, which have been associated with fluctuations in depressive symptoms (e.g.,

454 Hankin, 2010).

455 The pattern of correlations observed across cultures suggested that the nomological

456 network of the depression construct may differ depending on the studied population (Cronbach

$457 \&$ Meehl, 1955). For instance, although scores on BDI-II somatic-vegetative and cognitive-

458 affective symptoms were correlated among Egyptian participants, the relationship was not as

459 strong as that evidenced among Canadians. As suggested by Brouwer, Meijer, and Zevalkink

460 (2013), the existence of a strong association between somatic and cognitive symptoms among

461 Western individuals suggested that both subconstructs were not orthogonal and may be driven by

462 a higher order construct of depression. However, the differences in the strength of these

463 correlations may be attributable to the samples sizes between the cultural groups, whereby the

464 Canadian sample was slightly larger than the Egyptian sample. Nonetheless, it is possible that the

465 somatic and cognitive symptoms represent orthogonal constructs within the current sample of

466 Egyptians. Given the relatively simple design of this study and the modest sample size

467 employed, this hypothesis remains to be explored in the future.

This study extends previous research in many ways. First of all, this study employed a

469 secondary sample recruited from Canada to act as a cultural control group. Moreover, the use of 470 a non-depressed sample also provided additional baseline information. Second, special care and

471 attention went into the translation of measures used in this study (see Beshai et al., 2016, for

472 details regarding the translation process). As a testament to the success of the translation process,

473 the measures used in the current study possessed excellent and similar reliability estimates across

474 samples, and evidenced partial construct validity. Moreover, scalar equivalence analyses were 
475 conducted to ensure that the BDI-II scores were valid across cultures. By removing items that

476 displayed cultural variance, the differences found in this study were likely to reflect meaningful

477 and real cross-cultural differences in the construct of depression. The measures used herein are

478 globally used self-report measures of depression, and so the results obtained in this study can be

479 directly compared with a large body of literature arising from different parts of the world.

480 Despite these strengths, the study suffered from a number of limitations. First of all,

481 depressed Egyptians and Canadians were found to differ on a number of important

482 characteristics (age, socioeconomic status, depression scores), and so the observed differences in

483 symptom profiles may be due to these unexamined demographic or diagnostic differences.

484 However, using the same dataset, Beshai et al. (2016) found that, besides chronological age,

485 depressive symptoms and other depression-related constructs were not systematically related to

486 any of the examined demographic factors. Second, along with cut-offs on self-report measures,

487 the current study relied on a bespoke structured interview designed in accordance with DSM-IV

488 criteria for major depressive episode to ascertain depression diagnoses. As such, this interview

489 was not validated for this purpose. With this said, the interview items were reliable and

490 significantly predicted scores on self-report measures. Further, most "gold standard" interviews,

491 such as the Structured Clinical Interview for the DSM-IV (First et al., 1997), have never been

492 translated to Arabic, and so their use in this study was not feasible. Third, the current study relied

493 on scores of only one measure to examine self-reported differences across cultures in somatic

494 and cognitive symptoms. Fourth, the sample size in the current study was relatively modest. This

495 modest sample size makes generalizations to the population of Egyptian depression sufferers

496 difficult. Fifth, the sample recruited in Egypt for the purposes of this study may not be fully

497 representative of the general population of Egyptians. This is especially true given that all 
498 participants self-selected into the study, thus this may have skewed the presented results. Finally,

499 depression in this study was defined in accordance with DSM-IV criteria, which is a Western

500 conceptualization of the disorder. Accordingly, it is possible that Western definitions of the

501 depression do not map well onto this construct in Egypt, which may partially explain the

502 differential findings. With these limitations in mind, the present study still answered a number of

503 questions regarding self-reported symptoms of depression cross-culturally.

504 Given these limitations, future research should employ multiple methodologies (e.g.,

505 face-to-face interviews; self and clinician reports of symptomology) to examine the presentation

506 of somatic and cognitive features of depression in Egypt. Further, and considering cross-cultural

507 and gender differences obtained here, future research should employ larger samples of men and

508 women in order to replicate the findings of the present study. Depression and anxiety are often

509 comorbid and highly overlapping diagnostic construct (Clark \& Watson, 1991). Therefore, future

510 studies should examine whether there is agreement between reported symptom profiles of

511 anxiety and depression cross-culturally (Hoga et al., 2006).

\section{Conclusions}

513 Although both somatic and cognitive symptoms of depression are typically self-reported

514 among individuals of Egyptian descent, depressed Egyptian men may be reluctant to report

515 cognitive-affective symptoms in comparison to depressed men of Western descent. Further, it is

516 possible that cognitive and somatic symptoms represent orthogonal subconstructs of depression

517 in Egypt, and so it may be appropriate to measure these symptoms separately for individuals of

518 this culture. 


\section{References}

521 Addis, M. E., \& Cohane, G. H. (2005). Social scientific paradigms of masculinity and their implications for research and practice in men's mental health. Journal of Clinical Psychology, 61,1-15.

524

525

526

527

528

529

530

531

532

533

534

535

536

537

538

539

540

541

542

543

Addis, M. E. (2008). Gender and depression in men. Clinical Psychology: Science and Practice, 15,153-168.

Alansari, B. M. (2006). Internal consistency of an Arabic adaption of the Beck Depression Inventory-II with college students in eighteen Arab countries. Social Behaviour and Personality: An International Journal, 33, 503 - 212.

American Psychiatric Association (2000). Diagnostic and statistical manual of mental disorders (4h ed., text rev.). Washington, DC: Author.

Angst, J., Gamma, A., Gastapar, M., Lepine, J. P., Mendlewicz, J., \& Tylee, A. (2002). Gender differences in depression. European Archives of Psychiatry and Clinical Neuroscience, $252,201-209$.

Beck, A. T., Rush, A. J., Shaw, B. F., \& Emery, G. (1979). Cognitive therapy of depression. New York: The Guilford Press.

Beck, A. T., Steer, R. A., \& Brown, G. K. (1996). Manual for the beck depression inventory-II. San Antonio, TX: Psychological Corporation.

Beshai, S., Clark, C. M., \& Dobson, K. S. (2013). Conceptual and pragmatic considerations in the use of cognitive-behavioral therapy with Muslim clients. Cognitive Therapy and Research, 37, 197-206.

Beshai, S., Dobson, K. S., \& Adel, A. (2012). Cognition and dysphoria in Egypt and Canada: An examination of the cognitive triad. Canadian Journal of Behavioural Science, 44, 29-39. 
544 Beshai, S., Dobson, K. S., \& Adel, A. (2013). Psychometric properties of the Center for

545

546

547

548

549

550

551

552

553

554

555

556

557

558

559

560

561

562

563

564

565

Epidemiologic Studies Depression Scale in an Egyptian student sample: a preliminary report. Middle East Current Psychiatry, 20, 223-228.

Beshai, S., Dobson, K. S., Adel, A., \& Hanna, N. (2016) A cross-cultural study of the cognitive model of depression: Cognitive experiences converge between Egypt and Canada. PLOS ONE. e0150699.

Brouwer, D., Meijer, R. R., \& Zevalkink, J. (2013). On the factor structure of the Beck

Depression Inventory-II: G is the key. Psychological Assessment, 25, 136-145.

Burr, J., \& Chapman, T. (2004). Contextualizing experiences of depression in women from South Asian Communities: A discursive approach. Sociology of Health and Illness, 26, 433-452.

Byrne, B. M., Stewart, S. M., Kennard, B. D., \& Lee, P. W. H. (2007). The Beck Depression Inventory-II: Testing for measurement equivlanece and factor mean differences across Hong Kong and American adolescents. International Journal of Testing, 7, 293 - 309.

Canel-Cinarbas, D., Cui, Y., \& Lauridsen, E. (2011). Cross-cultural validation of the Beck Depression Inventory-II across U.S. and Turkish samples. Measurement and Evaluation in Counselling and Development, 44, 77 - 91.

Chentsova-Dutton, Y. E., \& Tsai, J. L. (2008). Understanding depression across cultures. In I. H. Gotlib \& C. L. Hammen (Eds.), Handbook of Depression (pp. 363-385). New York: Guildord Press.

Clark, L. A., \& Watson, D. (1991). Tripartite model of anxiety and depression: psychometric evidence and taxonomic implications. Journal of Abnormal Psychology, 100, 316. 
566 Cochran, S. V., Rabinowitz, F. E. (2000). Men and depression: Clinical and empirical 567 perspectives. San Diego, CA: Academic Press.

568 Cronbach, L. J., \& Meehl, P. E. (1955). Construct validity in psychological tests. Psychological $569 \quad$ Bulletin, 52, 281-302.

570 Dere, J., Sun, J., Zhao, Y., Persson, T. J., Zhu, X., Yao, S., ... Ryder, A. G. (2013). Beyond 571 "somatization" and "psychologization": Symptom-level variation in depressed Han 572 Chinese and Euro-Canadian outpatients. Frontiers in Psychology, 4, 377-390.

573

574

575

576

577

578

579

580

581

582

583

584

585

586

Dalgard, O. S., Dowrick, C., Lehtinen, V., Vazquez-Barquero, J. L., Casey, P., Wilkinson, G., Ayuso-Mateos, J. L., ... \& The ODIN Group. (2006). Negative life events, social support and gender differences in depression. Social Psychiatry and Psychiatric Epidemiology, $41,444-451$.

Dozois, D. J. A., Dobson, K. S., \& Ahnberg, J. L. (1998). A psychometric evaluation of the Beck Depression Inventory -II. Psychological Assessment, 10, 83 - 89.

Faul, F., Erdfelder, E., Lang, A.-G., \& Buchner, A. (2007). G*Power 3: A flexible statistical power analysis program for the social, behavioural, and biomedical sciences. Behaviour Research Methods, 39, 175-191.

First MB, Spitzer RL., Gibbon M, Williams JBW (1997) User's guide for the Structured Clinical Interview for DSM-IV Axis I Disorders. Washington, DC: American Psychiatric Press.

Goldberg D. P., \& Bridges, K. (1988). Somatic presentations of psychiatric illness in primary care setting. Journal of Psychosomatic Research, 32, 137-144. 
587 Hankin, B. L. (2010). Personality and depressive symptoms: Stress generation and cognitive 588 vulnerability to depression in a prospective daily diary study. Journal of Social Clinical Psychology, 29, 369-401.

590

591

592

593

594

595

596

597

598

599

600

601

602

603

604

605

606

607

Hoge, E. A., Tamrakar, S. M., Christian, K. M., Mahara, N., Nepal, M. K., Pollack, M. H., \& Simon, N. M. (2006). Cross-cultural differences in somatic presentation in patients with generalized anxiety disorder. Journal of Nervous and Mental Disease, 194, 962-966.

Hsu, L. G., Wan, Y. M., Chang, H., Summergrad, P., Tsang, B. P., \& Chen, H. (2008). Stigma of depression is more severe in Chinese Americans than Caucasian Americans. Psychiatry: Interpersonal And Biological Processes, 71, 210-218.

IBM Corp. Released 2010. IBM SPSS Statistics for Windows, Version 19.0. Armonk, NY: IBM Corp.

Kandil, H. M. (2005). Study risk factors predicting the comorbidity between bipolar disorder and anxiety disorders in a sample of Egyptian patients [dissertation]. Alexandria: University of Alexandria.

Kalibatseva, Z., Leong, F. T. L., \& Ham, E. H. (2014). A symptom profile of depression among Asian Americans: is there evidence for differential item functioning of depressive symptoms? Psychological Medicine, 44, 2567-2578.

Kalibatseva, Z., \& Leong, F. T. L. (2011). Depression among Asian Americans: Review and recommendations. Depression Research and Treatment. 320902.

Karasz, A. (2005). Cultural differences in conceptual models of depression. Social Science \& Medicine, 60, 1625-1635. 
608 Kessler, R. C., \& Ustun, T. B. (2004). The World Mental Health (WMH) survey initiative 609 version of the World Health Organization (WHO) composite international diagnostic 610 interview (CIDI). International Journal of Methods in Psychiatric Research, 13, 93-121.

611 Koenig, H. G., Cohen, H. J., Blazer, D. G., Kudler, H. S., Krishnan, K. R. R., \& Sibert, T. E. (1995). Religious coping and cognitive symptoms of depression in elderly medical patients. Psychosomatics, 36, 369-375.

614 Kornstein, S. G., Schatzberg, A. F., Thase, M. E., Yonkers, K. A., McCullough, J. P., Keitner, G. 615 I., Gelenberg, A. J., ... \& Keller, M. B. (2000). Gender differences in chronic major and 616 double depression. Journal of Affective Disorder, 60, 1-11.

617 Koss-Chioino, J. D. (1999). Depression among Puerto Rican women: Culture, etiology and 618 diagnosis [Special issue]. Hispanic Journal of Behavioural Sciences, 21, 330-350.

619 Kuehner, C. (2003). Gender differences in unipolar depression: An update of epidemiological 620 findings and possible explanations. Acta Psychiatrica Scandinaiva, 108, 163 - 174.

621 Lam, K., Marra, C., \& Salzinger, K. (2005). Social reinforcement of somatic versus psychological description of depressive events. Behaviour Research and Therapy, 43,

624 Lee, S., \& Kleinman, A. (2007). Are somatoform disorders changing with time? The case of 625 neurasthenia in China. Psychosom Med, 69, 846-9.

Leff, J. (1988). Psychiatry around the globe: A transcultural view. London, England: Gaskell/Royal College of Psychiatrists. edition across race with South African university students. South African Journal of Psychology: 0081246316645045. 
631 Matthey, S., Barnett, B. E. W., \& Elliott, A. (1997). Vietnamese and Arabic women's responses

632 to the diagnostic interview schedule (depression) and self-report questionnaires: Cause 633 for concern. Australasian Psychiatry, 31, 360-369.

634 Meredith, W. (1993). Measurement invariance, factor analysis and factorial invariance. 635 Psychometrika, 58, 525-543.

636 Moieni, M., Irwin, M. R., Jevtic, I., Olmstead, R., Breen, E. C., \& Eisenberger, N. I. (2015). Sex 637 differences in depressive and socioemotional responses to an inflammatory challenge: 638 Implications for sex differences in depression. Neuropsychopharmacology, 40, 1709$639 \quad 1716$.

640 Nolen-Hoeksema, S., \& Jackson, B. (2001). Mediators of the gender differences in rumination. $641 \quad$ Psychology of Women Quarterly, 25, $37-47$.

642 Okasha, A. (1999). Mental health in the Middle East: An Egyptian perspective. Clinical 643 Psychology Review, 19, 917-933.

644 Parker, G., Cheah, Y. C., \& Roy, K. (2001). Do the Chinese somatize depression? A cross645 cultural study. Social Psychiatry and Psychiatric Epidemiology, 36, 287-293.

646 Radloff, L. S. (1977). The CES-D scale: A self-report depression scale for research in the general 647 population. Applied Psychological Measurement, 1, 385.

648 Ryder, A. G., \& Chentsova-Dutton, Y. E. (2012). Depression in cultural context: “Chinese 649 somatization," revisited. Psychiatric Clinics of North America, 35, 15-36.

650 Ryder, A. G., Yang, J., \& Heine, S. J. (2002). Somatization vs. psychologization of emotion 651 distress: A paradigmatic example for cultural psychopathology. Online Readings in $652 \quad$ Psychology and Culture. 10(2). 
653 Ryder, A. G., Yang, J., Zhu, X., Yao, S., Yi, H., Heine, S. J., \& Bagby, R. M. (2008). The

654 cultural shaping of depression: somatic symptoms in China, psychological symptoms in 655 North America? Journal of Abnormal Psychology, 117, 300-313.

656 Sami, M., \& El-Gawad, A. (1995). Transcultural psychiatry in Egypt. In I. Al-Issa (Ed),

657 Handbook of culture and mental illness: An international perspective (pp. 53-64).

658 Connecticut: International Universities Press, Inc.

659 Schuch, J. J. J., Roest, A. M., Nolen, W. A., Penninx, B. W. J. H., \& de Jonge, P. (2014). Gender

660 differences in major depressive disorder: Results from the Netherlands study of

661 depression and anxiety. Journal of Affective Disorders, 156, 156 - 163.

662 Silverstein, B. (2002). Gender differences in the prevalence of somatic versus pure depression: A 663 replication. The American Journal of Psychiatry, 159, 1051 - 1052.

664 Simon, G. E., VonKorf, M., Piccinelli, M., Fullerton, C., \& Ormel, J. (1999). An international 665 study of the relation between somatic symptoms and depression. The New England $666 \quad$ Journal of Medicine, 341, 1329-1335.

667 Slocum, S. L., Gelin, M. N., \& Zumbo, B. D. (2003). Statistical and graphical modeling to 668 investigate differential item functioning for rating scale and Likert item formats.

669 Developments in the theories and applications of measurement, evaluation, and research 670 methodology across the disciplines, 1.

671 Tylee, A., \& Gandhi, P. (2005). The importance of somatic symptoms in depression in primary care. Primary Care Companion to The Journal of Clinical Psychiatry, 7, 167-176.

673 Whisman, M. A., Judd, C. M., Whiteford, N. T., \& Gelhorn, H. L. (2012). Measurement invariance of the Beck Depression Inventory-Second edition (BDI-II) across gender, race, 675 and ethnicity in college students. Assessment, 20, $419-428$. 
676 Yao, Z., Yan, R., Wei, M., Tang, H., Qin, J., \& Lu, Q. (2014). Gender differences in brain

677 activity and the relationship between brain activity and differences in prevalence rates

678 between male and female major depressive disorder patients; A resting-state fMRI study.

$679 \quad$ Clinical Neurophysiology, 125, 2232 - 2239.

680 Zhou, X., Dere, J., Zhu, X., Yao, S., Chentsova-Dutton, Y. E., \& Ryder, A. G. (2011). Anxiety

681 symptom presentations in Han Chinese and Euro-Canadian outpatients: Is distress always 682 somatised in China? Journal of Affective Disorders, 135, 111 - 114.

683 Zimmerman, M., \& Mattia, J. I. (2001). A self-report scale to help make psychiatric diagnoses:

684 the Psychiatric Diagnostic Screening Questionnaire. Archives of General Psychiatry, 58, $685 \quad 787-794$

686 Zumbo, B. D. (1999). A handbook on the theory and methods of differential item functioning 687 (DIF): Logistic regression modeling as a unitary framework for binary and Likert-type 688 (ordinal) item scores. Ottawa, Ontario, Canada: Directorate of Human Resources 689 Research and Evaluation, Department of National Defense.

690

691 


\section{Table $\mathbf{1}$ (on next page)}

Table 1. Sample characteristics of the Egyptian and Canadian samples

* New calculated BDI-II total scores with cultural variant items removed. 
1

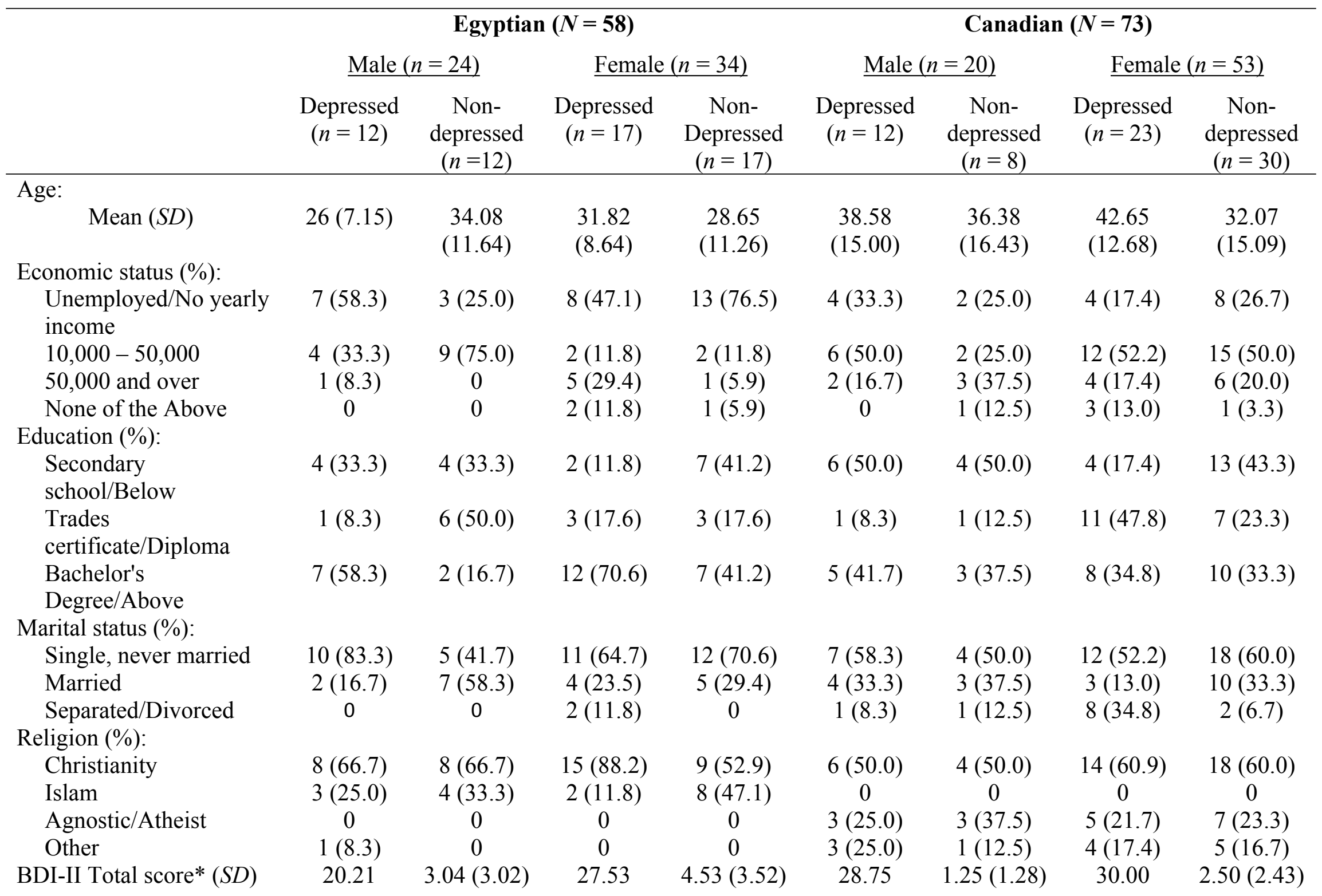




\begin{tabular}{|c|c|c|c|c|c|c|c|c|}
\hline & $(6.52)$ & & $(8.38)$ & & (10.17) & & $(8.53)$ & \\
\hline $\begin{array}{l}\text { BDI-II Somatic-vegetative } \\
\text { symptoms* }\end{array}$ & $\begin{array}{l}11.96 \\
(4.39)\end{array}$ & $1.21(1.34)$ & $\begin{array}{l}13.59 \\
(5.07)\end{array}$ & $2.35(3.10)$ & $\begin{array}{l}14.42 \\
(5.63)\end{array}$ & $0.75(0.89)$ & $\begin{array}{l}16.57 \\
(4.03)\end{array}$ & 1.57 (1.59) \\
\hline $\begin{array}{l}\text { BDI-II Cognitive-affective } \\
\text { symptoms* }\end{array}$ & $\begin{array}{c}8.25 \\
(4.02)\end{array}$ & $1.83(2.21)$ & $\begin{array}{l}13.94 \\
(4.59)\end{array}$ & $2.18(3.52)$ & $\begin{array}{l}14.33 \\
(5.26)\end{array}$ & $0.50(0.53)$ & $\begin{array}{l}13.43 \\
(5.14)\end{array}$ & $0.93(1.53)$ \\
\hline
\end{tabular}




\section{Figure 1}

Figure 1.Three-way interactions of BDI-II cognitive-affective subscale scores.

A) Depressed individuals. Covarying for age and somatic-vegetative subscale scores; and B) non-depressed individuals. Covarying for age and somatic-vegetative subscale scores. 
a)

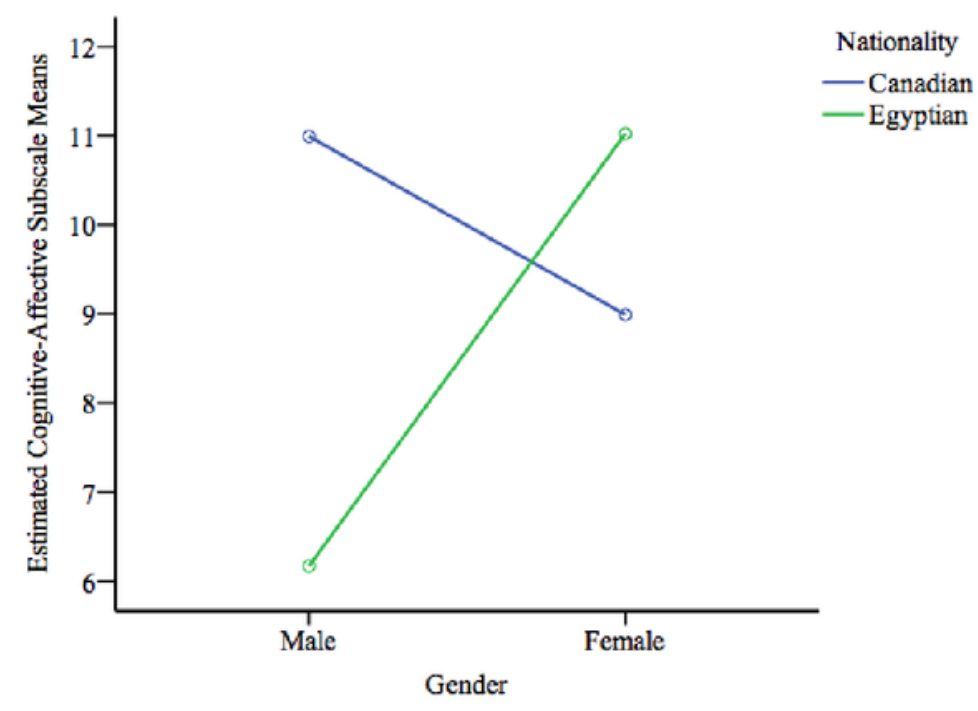

b)

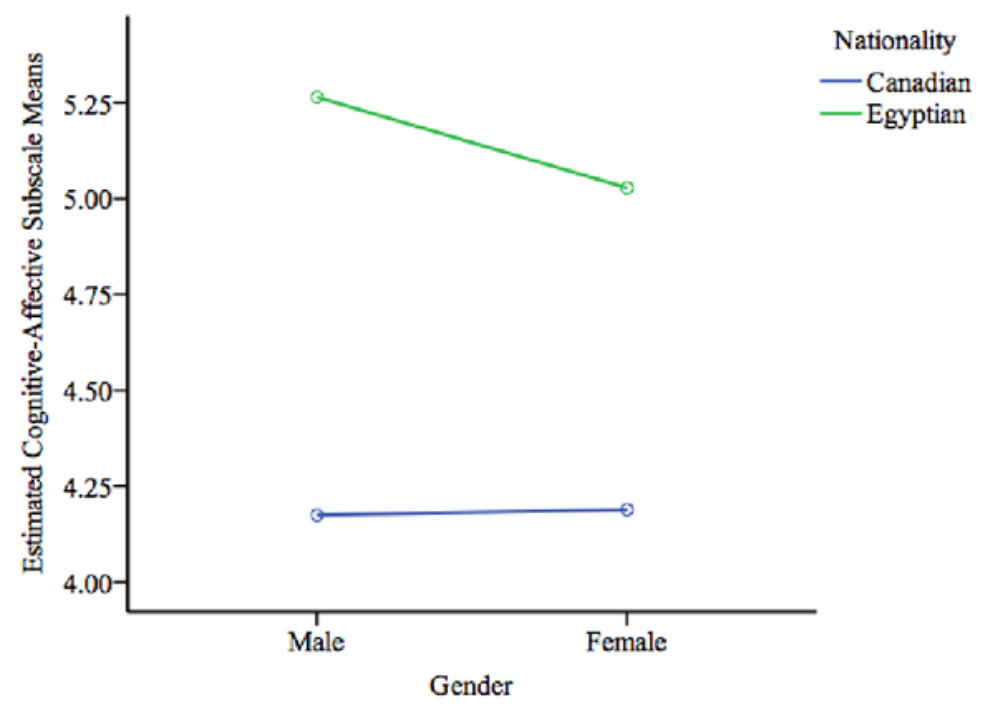

